\title{
Construction and Validation of a Nomogram for Predicting the Risk of Deep Vein Thrombosis in Hepatocellular Carcinoma Patients After
} Laparoscopic Hepatectomy: A Retrospective Study

\section{Yao Chen* \\ Jianping Zhao* \\ Zhanguo Zhang (D) \\ Zeyang Ding $(\mathbb{D}$ \\ Yifa Chen \\ Xiaoping Chen \\ Wanguang Zhang}

Hepatic Surgery Center, Tongji Hospital, Tongji Medical College, Huazhong University of Science and Technology (HUST), Wuhan, People's Republic of China

*These authors contributed equally to this work
Correspondence: Yifa Chen;

Wanguang Zhang

Hepatic Surgery Center, Tongji Hospital,

Tongji Medical College, HUST, 1095

Jiefang Avenue, Wuhan, Hubei Province,

430030, People's Republic of China

Email yfchen4533@163.com;

wgzhang@tjh.tjmu.edu.cn
Background: The incidence of deep vein thrombosis (DVT) in hepatocellular carcinoma (HCC) patients after laparoscopic hepatectomy $(\mathrm{LH})$ is unclear, and there is no effective method for DVT risk assessment in these patients.

Methods: The data from the total of 355 consecutive HCC patients who underwent LH were included. A DVT risk algorithm was developed using a training set (TS) of 243 patients, and its predictive performance was evaluated in both the TS and a validation set (VS) of 112 patients. The model was then used to develop a DVT risk nomogram (TRN).

Results: The incidence of DVT in the present study was $18.6 \%$. Age, sex, body mass index (BMI), comorbidities and operative position were independent risk factors for DVT in the TS. The model based on these factors had a good predictive ability. In the TS, it had an area under the receiver operating characteristic (AUC) curve of 0.861 , Hosmer-Lemeshow (H-L) goodness of fit $p$ value of 0.626 , sensitivity of $44.4 \%$, specificity of $96.5 \%$, positive predictive value (PPV) of $74.1 \%$, negative predictive value (NPV) of $88.4 \%$, and accuracy of $86.8 \%$. In the VS, it had an AUC of 0.818 , H-L $p$ value of 0.259 , sensitivity of $38.1 \%$, specificity of $98.9 \%$, PPV of $88.9 \%$, NPV of $87.4 \%$, and accuracy of $87.5 \%$. The TRN performed well in both the internal and the external validation, indicating a good clinical application value. The TRN had a better predictive value of DVT than the Caprini score $(p<$ $0.001)$.

Conclusion: The incidence of DVT after LH was high, and should not be neglected in HCC patients. The TRN provides an efficacious method for DVT risk evaluation and individualized pharmacological thromboprophylaxis.

Keywords: deep vein thrombosis, hepatocellular carcinoma, laparoscopic hepatectomy, nomogram

\section{Introduction}

Venous thromboembolism (VTE), including deep vein thrombosis (DVT) and pulmonary embolism (PE), is a common complication and second leading cause of death in cancer patients. ${ }^{1}$ A study based on the American College of Surgeons National Surgical Quality Improvement Program (NSQIP) database showed that the incidence of symptomatic VTE after hepatectomy was $2.9 \%{ }^{2}$ Several studies from Asia indicated that the incidence of perioperative VTE was between 14 and 57 per 100,000 people per year and varied from region to region. ${ }^{3-5}$ Although the apparent 
incidence of VTE in previous studies was not high, we hypothesized that asymptomatic VTE cases may often be overlooked. The available data suggested that the rate of distal DVT spreading to the popliteal vein or higher veins was $17.0 \%$, with up to $5.8 \%$ progressing to $\mathrm{PE}$ without intervention. ${ }^{6}$ Numerous studies investigated potential risk factors for VTE, ${ }^{7-10}$ but the morbidity and risk factors of DVT after laparoscopic hepatectomy (LH) in hepatocellular carcinoma (HCC) patients remain unknown. ${ }^{11}$

Although laparoscopic surgery represents a significant improvement of surgical technique, it nevertheless comes with its own challenges and intraoperative adverse events may lead to an increase of perioperative complications. ${ }^{12}$ For example, Sharma et al reported that pneumoperitoneum and placing the patient in reverse Trendelenburg position resulted in venous stasis in lower extremities, ${ }^{13}$ potentially leading to perioperative DVT and PE. ${ }^{14}$ To our best knowledge, there are no studies focusing on the relationship between $\mathrm{LH}$ and postoperative DVT.

The Caprini score is widely used in surgical departments to estimate the risk of DVT, and has been validated in over 250,000 patients in more than 100 clinical trials worldwide. $^{15}$ Although the Caprini score provides an important reference for surgeons, it does not perform very well in stratifying $\mathrm{HCC}$ patients after hepatectomy, ${ }^{7}$ and there are few effective tools for predicting the DVT risk of HCC patients after LH. The purpose of the present study was to assess the incidence of DVT and identify the relative risk factors in order to develop and validate a DVT risk nomogram (TRN) for HCC patients after LH.

\section{Methodology}

\section{Patients}

A retrospective study was conducted on consecutive $\mathrm{HCC}$ patients who underwent LH in our center from June 2016 to January 2020. The inclusion criteria were: (1) LH and no conversion; (2) HCC diagnosed pathologically and no distant metastasis; (3) no previous treatment for HCC; (4) no history of other malignancies; (5) no history of VTE or vascular disease (for example, tube in blood vessel, heart disease, lung disease, leg swelling or visible varicose veins); (6) no perioperative pharmacological thromboprophylaxis; (7) no acute infection, recent orthopedic surgery, serious trauma or stroke; (8) no recent pregnancy, birth or miscarriage; (9) lower extremity color Doppler flow imaging examination within 3 days after operation; and (10) detailed and precise follow-up records. The patients from
June 2016 to October 2018 were assigned to the Training Set (TS) and the others to the Validation Set (VS). The written informed consent for data use was obtained from all the patients. This study was approved by the Ethics Committee of Tongji Hospital, and was performed in accordance with the Declaration of Helsinki. ${ }^{16}$

\section{Surgery Techniques}

The type of hepatectomy was decided based on the tumor and liver condition for each patient, as described previously. ${ }^{17}$ The reverse Trendelenburg position was routinely applied, but the lateral position was used for right hepatectomy. Pneumoperitoneum pressure was maintained at $10-12 \mathrm{mmHg}$, and the Pringle maneuver was performed during surgery if necessary. Major hepatectomy was defined as the resection of three or more Couinaud segments, while minor hepatectomy was defined as the resection of fewer than three Couinaud segments. Portal hypertension was defined as the presence of either esophageal varices or coexistence of splenomegaly with platelet counts $<100 \times 10^{9} /$ L. $^{18,19}$

\section{Definition and Management of DVT}

Proximal DVT was defined based on a thrombus present in the popliteal or more proximal veins, and distal DVT was based on a thrombus present in the calf veins, ${ }^{20}$ and diagnosed by lower extremity color Doppler flow imaging examination on the second or third day after surgery. Patients with both proximal and distal DVT were classified as having proximal DVT. Computed tomographic pulmonary arteriography was performed when the patient presented suspicious symptoms of PE. ${ }^{21-23}$ All patients were subjected to intermittent pneumatic compression since the first day after surgery until ambulation, and were encouraged to accomplish early mobilization after surgery. The measures of early mobilization in our center were as follows: the patients are encouraged to accomplish mobilization (for example, sitting up and moving limbs) on the bed by postoperative day 1, and get out of bed and walk by postoperative day 2 . Anticoagulant therapy was performed in patients with DVT confirmed by imaging and no significant bleeding risk. The therapeutic scheme of DVT was as follows: During hospitalization, the patients received low molecular weight heparin (LMWH), $4.25 \mathrm{kU}$, subcutaneously, once a day and were examined by lower extremity color Doppler flow imaging every five days until the DVT disappeared. After discharge, the DVT patients were administered Rivaroxaban, $15 \mathrm{mg}$ twice daily for the first 
21 days, and then switched to $20 \mathrm{mg}$ daily (creatinine clearance $\geq 30 \mathrm{~mL} / \mathrm{min}$ ), ${ }^{24}$ and the lower extremity color Doppler flow imaging examination was performed once a month until the DVT disappeared. Multidisciplinary treatment was implemented if necessary. All the DVT patients received anticoagulant therapy and were followed up for at least 3 months. $^{25}$

\section{Statistical Analysis}

All the data were analyzed using SPSS 22.0 (IBM Corporation, Armonk, NY, USA) and R version 3.6.3 (R Foundation for Statistical Computing, Vienna, Austria). Continuous variables were expressed as means \pm standard deviations (SD) and categorical variables were expressed as numbers and percentages. Continuous variables were compared using the Mann-Whitney $U$-test, while categorical variables were compared using Pearson's chi-squared or Fisher's exact test (2-tailed). The significant variables in the univariate analysis were used for the binary logistic regression model to generate an algorithm for predicting the risk of DVT in the TS. Forward stepwise variable selection was applied to build the model with the Akaike information criterion (AIC) as the stopping rule. The area under the receiver operating characteristic curve (AUC) and the Hosmer-Lemeshow (H-L) goodness of fit test were used to assess the predictive ability of the model in both the TS and the VS The De Long test was used to compare the AUCs. The sensitivity, specificity, positive predictive value (PPV), negative predictive value (NPV) and accuracy were calculated. The TRN was created in the TS using the "rms" R package (Appendix A1). Calibration curves based on 1000 bootstrap resampling iterations were used to examine the performance of the TRN in both the TS (internal validation) and the VS (external validation). Finally, the decision curve was plotted to evaluate the clinical application value of the TRN based on the net benefit at different risk thresholds. Differences with $p<0.05$ were considered statistically significant.

\section{Results}

\section{Clinicopathological Characteristics}

The workflow was summarized in Figure 1. A total of 355 consecutive patients were recruited in this study (TS: $\mathrm{n}=$ 243 , including 45 with and 198 without; VS: $n=112,21$ with and 91 without DVT). A total of $66(18.6 \%)$ patients were diagnosed with DVT, including $40(60.6 \%)$ with unilateral distal DVT, 24 (36.4\%) with bilateral distal DVT and 2
(3.0\%) with unilateral proximal DVT. Two patients in the TS had ipsilateral proximal and distal DVT and were classified as having unilateral proximal DVT (Appendix A2). No PE occurred. The clinicopathological features of the patients are listed in Table 1 . There were fewer elderly $(112,31.5 \%)$ and more male $(286,80.6 \%)$ patients. A total of $63(17.7 \%)$ patients had a body mass index (BMI) of more than $25 \mathrm{~kg} /$ $\mathrm{m}^{2}$. A total of $75(21.1 \%)$ and $258(72.7 \%)$ patients had comorbidities (diabetes and/or hypertension) and viral hepatitis (HBV or HCV), respectively. More than half of the patients $(228,64.2 \%)$ had a hematocrit grade $\geq 39$. The mean platelet count (PLT) and international normalized ratio (INR) were $167.3 \pm 51.9 \times 10^{9} / \mathrm{L}$ and $1.1 \pm 0.1$, respectively. A total of $60(16.9 \%)$ patients had portal hypertension. Most of the patients had solitary tumors $(303,85.4 \%)$, and the maximum tumor size (MTS) was $4.3 \pm 2.9 \mathrm{~cm}$. According to the Barcelona clinic liver cancer (BCLC) scale, $326(91.8 \%)$ patients had stage A disease, and 251 (70.7\%) patients received minor hepatectomy. Lateral position during the operation was used in 133 (37.5\%) patients and the mean operation time (OT) was $5.5 \pm 1.7$ hours. There were 95 (26.8\%) patients who needed intraoperative transfusion (IT) and 19 (5.4\%) patients received hemostatic therapy. In the present study, the incidence of complications was $27.3 \%$, and $18(5.1 \%)$ patients had major postoperative complications (Clavien-Dindo classification $\geq 3$ ). All patients recovered well and no deaths occurred due to surgery. The clinicopathological features of patients did not exhibit significant differences between the two sets.

\section{Development of the Risk Score Algorithm}

Univariate analysis showed that age, sex, BMI, comorbidities, hematocrit grade, IT and operative position (OPP) were associated with DVT in the TS. However, only age, sex, BMI, comorbidities and OPP remained independent risk factors in multivariate analysis, and were consequently applied to develop a risk score algorithm for predicting DVT (Table 2). We then compared the clinicopathological features of the patients with and without DVT (Appendix A3). The factors associated with DVT in the TS according to univariate analysis also exhibited significant differences between the patients with and without DVT, except for the use of hemostatics $(p=0.036)$, which was excluded in the multivariate analysis. We further assessed the predictive performance of the algorithm in both the TS and the VS. 


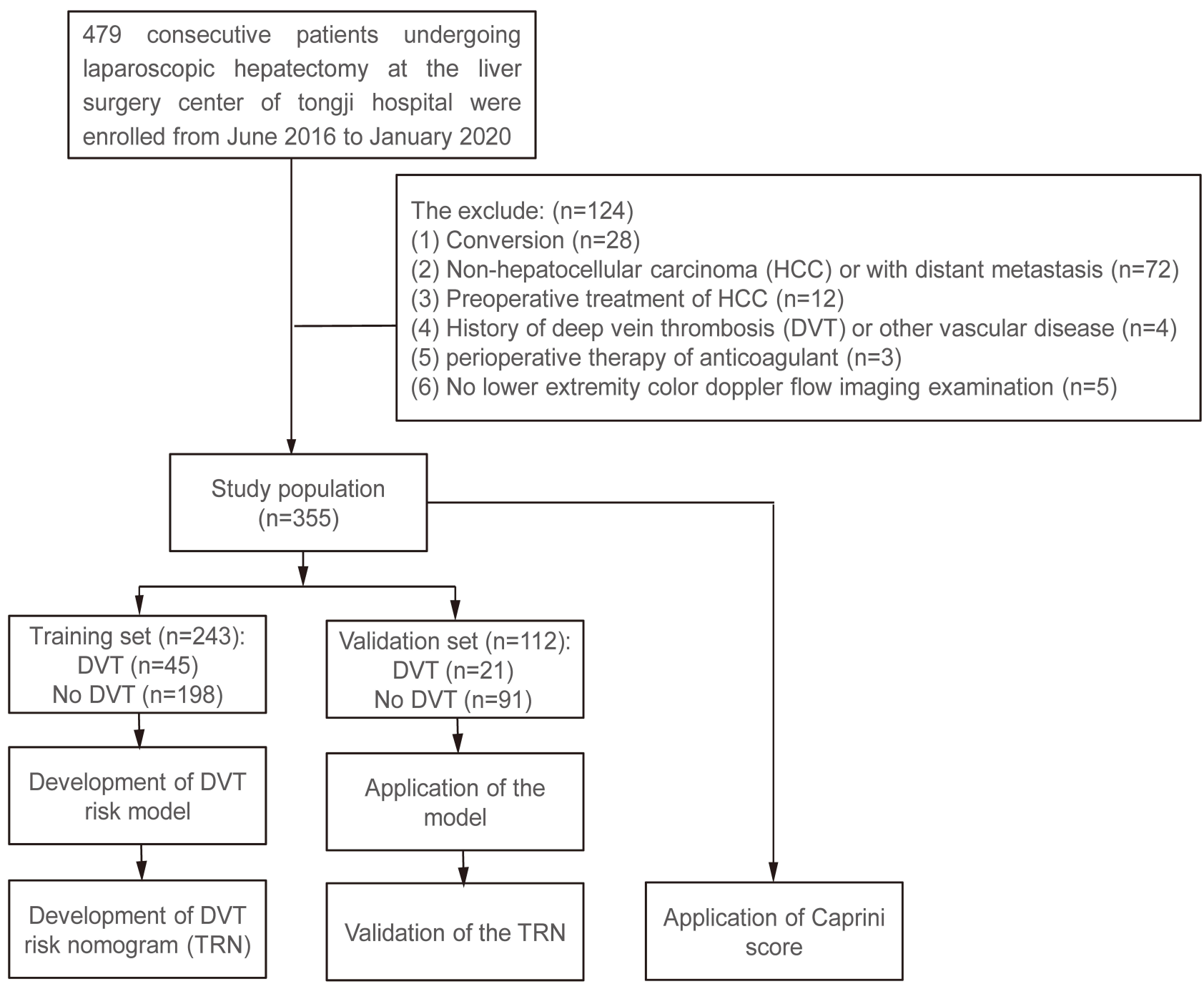

Figure I Flowchart of study design.

In the TS, the model had an AUC of 0.861 with a $95 \%$ confidence interval (CI) of $0.802-0.920 \%$, a $\mathrm{H}-\mathrm{L}$ test $p$ value of 0.626 , sensitivity of $44.4 \%$ with a $95 \%$ CI of $30.0 \%-59.9 \%$, specificity of $96.5 \%$ with a $95 \%$ CI of $92.6 \%-98.4 \%$, PPV of $74.1 \%$ with a $95 \%$ CI of $53.4 \%$ $88.1 \%$, NPV of $88.4 \%$ with a $95 \%$ CI of $83.2 \%-92.2 \%$, and accuracy of $86.8 \%$ with a $95 \%$ CI of $82.5 \%-91.1 \%$. In the VS, the model had an AUC of 0.818 with a $95 \%$ CI of $0.716-0.919$, a H-L test $p$ value of 0.259 , sensitivity of $38.1 \%$ with a $95 \%$ CI of $19.0 \%-61.3 \%$, specificity of $98.9 \%$ with a $95 \%$ CI of $93.2 \%-99.9 \%$, PPV of $88.9 \%$ with a $95 \%$ CI of $50.7 \%-99.4 \%$, NPV of $87.4 \%$ with a $95 \%$ CI of $79.0 \%-92.8 \%$, and accuracy of $87.5 \%$ with a $95 \%$ CI of $81.4-93.6 \%$ (Appendix A4, Figure 2). There was no significant difference of the AUC between the two sets $(p=0.474)$.

\section{Predictive Nomogram for the Risk of DVT}

We developed a TRN for simpler and more intuitive clinical application (Figure 3A). According to the calibration curve (Figure 3B and C), the TRN performed well with the ideal model in both tested datasets. Furthermore, the TRN added a net benefit over either the "treat all" or the "treat none" scheme in the decision curve (Figure 4).

\section{Application of the Caprini Score}

We assessed the ability of the Caprini score to predict DVT after LH in HCC patients. However, we found that all the patients scored $\geq 5$ and were defined as having a high risk of DVT (Appendix A5) ${ }^{26}$ The AUC of the Caprini score was 0.610 , which was significant lower than 
Table I Clinicopathological Features of the Patients

\begin{tabular}{|c|c|c|c|c|}
\hline Variables & Total $n=355$ & TS $n=243$ & VS $n=112$ & $p$ \\
\hline Age (years) $(\geq 60)$ & $112(31.5)$ & $79(32.5)$ & $33(37.5)$ & 0.357 \\
\hline Sex (male) & $286(80.6)$ & $190(78.2)$ & $96(85.7)$ & 0.096 \\
\hline BMI $\left(\mathrm{kg} / \mathrm{m}^{2}\right)(>25)$ & $63(17.7)$ & $45(18.5)$ & $18(16.1)$ & 0.575 \\
\hline Comorbidities (yes) & $75(21.1)$ & $47(19.3)$ & $28(25.0)$ & 0.225 \\
\hline Virus hepatitis (yes) & $258(72.7)$ & $183(75.3)$ & $75(67.0)$ & 0.101 \\
\hline Hematocrit grade $(\geq 39)$ & $228(64.2)$ & $153(63.0)$ & $75(67.0)$ & 0.465 \\
\hline PLT $\left(\times 10^{9} / \mathrm{L}\right)$ & $167.3 \pm 51.9$ & $166.2 \pm 52.7$ & $169.6 \pm 50.3$ & 0.604 \\
\hline INR & $1.1 \pm 0.1$ & $1.1 \pm 0.1$ & $1.0 \pm 0.1$ & 0.060 \\
\hline Portal hypertension (yes) & $60(16.9)$ & $4 \mid(16.9)$ & $19(17.0)$ & 0.983 \\
\hline Tumor number (multiple) & $52(14.6)$ & $37(15.2)$ & $15(13.4)$ & 0.650 \\
\hline MTS (cm) & $4.3 \pm 2.9$ & $4.2 \pm 2.8$ & $4.4 \pm 3.2$ & 0.795 \\
\hline BCLC stage $(A)$ & $326(91.8)$ & $223(91.8)$ & $103(92.0)$ & 0.950 \\
\hline Extent of hepatectomy (major) & $104(29.3)$ & $70(28.8)$ & $34(30.4)$ & 0.573 \\
\hline OPP (lateral position) & $133(37.5)$ & $91(37.5)$ & $42(37.5)$ & 0.993 \\
\hline OT (hour) & $5.5 \pm 1.7$ & $5.4 \pm 1.6$ & $5.7 \pm 1.8$ & 0.463 \\
\hline IT (yes) & $95(26.8)$ & $67(27.6)$ & $28(25.0)$ & 0.611 \\
\hline Hemostatics (yes) & $19(5.4)$ & $14(5.8)$ & $5(4.5)$ & 0.614 \\
\hline Complication grade & $97(27.3)$ & $70(28.8)$ & $27(24.1)$ & 0.564 \\
\hline$\geq 3$ & $18(5.1)$ & $12(4.9)$ & $6(5.4)$ & \\
\hline$<3$ & 79 (22.2) & $58(23.9)$ & $21(18.7)$ & \\
\hline
\end{tabular}

Abbreviations: BMI, body mass index; PLT, platelets; INR, international normalized ratio; MTS, maximum tumor size; BCLC, Barcelona clinic liver cancer; OPP, operative position; OT, operative time; IT, intraoperative transfusion; TS, training set; VS, validation set.

that of the TRN developed in the present study $(p<0.001$ vs the TS; $p=0.001$ vs the VS) (Figure 2). What's more, the decision curve of the Caprini score was closed to the curves of "treat all" and "treat none", which means that almost no net benefit could be added by the Caprini score for predicting the risk of DVT (Figure 4).

\section{Follow-Up and Outcomes of DVT Patients}

All the DVT patients received anticoagulant therapy for at least 3 months. DVT disappeared within 1 month in 57 (86.4\%) cases, with chronic DVT remaining in 9 (13.6\%) cases, including 3 unilateral distal, 5 bilateral distal and 1 unilateral proximal DVT case. ${ }^{27}$ In another 6 (9.1\%) cases, the DVT disappeared in the next two months, while 2 $(3.0 \%)$ unilateral distal DVT patients and 1 (1.5\%) bilateral distal DVT patient still had the thrombus after 3 months (Table 3). No PE or major bleeding occurred during the treatment.

\section{Discussion}

There is increasing evidence that VTE is a significant cause of morbidity and mortality during the postoperative period and the second leading cause of death in cancer patients. ${ }^{1,28,29}$ Several studies have found VTE rates after hepatectomy ranging from 2.4 to $4.6 \%{ }^{28,30,31}$ However, we should pay attention to the fact that asymptomatic VTE cases may often be overlooked. Although the Caprini score, widely used in surgical departments, provides an important reference for surgeons, ${ }^{15}$ it has limitations when assessing the DVT risk of HCC patients after liver surgery. ${ }^{7}$ Moreover, the morbidity and risk factors of DVT in HCC patients after LH remain unknown. 
Table 2 Factors Associated with the DVT in the TS

\begin{tabular}{|c|c|c|c|c|}
\hline \multirow[t]{2}{*}{ Variable } & \multicolumn{2}{|c|}{ Univariate Analysis } & \multicolumn{2}{|c|}{ Multivariate Analysis } \\
\hline & OR $(95 \% \mathrm{Cl})$ & $p$ & OR (95\% Cl) & $p$ \\
\hline Age $(\geq 60$ vs $<60)$ & $5.153(2.56 \mid-10.366)$ & $<0.001$ & $3.031(1.342-7.039)$ & 0.008 \\
\hline Sex (female vs male) & $2.65 I(1.368-5.136)$ & 0.004 & 13.966(5.486-39.438) & $<0.001$ \\
\hline BMI (>25 vs $\leq 25)$ & $3.215(1.555-6.648)$ & 0.002 & $4.219(1.553-11.858)$ & 0.005 \\
\hline Comorbidities (yes vs no) & $4.437(2.173-9.060)$ & $<0.001$ & $9.033(3.654-23.966)$ & $<0.001$ \\
\hline Virus hepatitis (yes vs no) & $0.670(0.329-1.366)$ & 0.670 & & \\
\hline Hematocrit grade ( $\geq 39$ vs $<39$ ) & $0.349(0.180-0.677)$ & 0.002 & & \\
\hline PLT, $\times 10^{9} / \mathrm{L}$ & $1.003(0.997-1.009)$ & 0.306 & & \\
\hline INR & $0.988(0.018-53.26 \mathrm{I})$ & 0.995 & & \\
\hline Portal hypertension (yes vs no) & $1.539(0.691-3.427)$ & 0.291 & & \\
\hline Tumor No (multiple vs single) & $0.648(0.238-1.769)$ & 0.398 & & \\
\hline MTS & $0.913(0.79 \mid-1.054)$ & 0.213 & & \\
\hline BCLC tumor stage ( $B$ vs $A$ ) & $0.465(0.104-2.08 I)$ & 0.317 & & \\
\hline $\begin{array}{l}\text { Extent of hepatectomy (major vs } \\
\text { minor) }\end{array}$ & $0.657(0.306-1.413)$ & 0.282 & & \\
\hline OPP (lateral vs supine) & $3.529(1.801-6.916)$ & $<0.001$ & $3.648(I .58 \mid-8.837)$ & 0.003 \\
\hline OT & $0.875(0.704-1.087)$ & 0.228 & & \\
\hline IT (yes vs no) & $2.284(1.163-4.485)$ & 0.016 & & \\
\hline Hemostatics (yes vs no) & $1.834(0.548-6.137)$ & 0.325 & & \\
\hline Complication grade $(\geq 3$ vs $<3$ ) & $1.500(0.389-5.779)$ & 0.556 & & \\
\hline
\end{tabular}

Abbreviations: TS, training set; DVT, deep vein thrombosis; BMI, body mass index; PLT, platelets; INR, international normalized ratio; BCLC, Barcelona clinic liver cancer; Tumor No, tumor number; MTS, maximum tumor size; OT, operative time; OPP, operative position; IT, intraoperative transfusion; OR, odds ratio; Cl, confidence interval.

In the present study, we assessed the incidence of DVT in HCC patients after LH. Considering the high potential risk of VTE in HCC patients after hepatectomy, lower extremity color Doppler flow imaging was recommended within 3 days after surgery. As a result, 66 (18.6\%) patients were diagnosed with DVT after LH, and all the DVT cases were asymptomatic. The incidence of DVT in the present study was much higher than what was found in previous studies, but it was in agreement with the results of a prospective study in Japan, with an incidence of $24.3 \%{ }^{31,32}$ The studies with low incidence of VTE were retrospective and the diagnostic VTE examination was more likely to be performed in patients with certain symptom or indications of VTE. Furthermore, the patients in the present study all had HCC, and $72.7 \%$ also had viral hepatitis. Studies have demonstrated that malignant tumors and chronic liver disease were related to hypercoagulability and a high risk of VTE. ${ }^{33,34}$ What's more, all the patients received LH, whereby the $\mathrm{CO}_{2}$ pneumoperitoneum pressure could decrease cardiac output and increase peripheral vascular resistance, thus affected tissue perfusion and leading to damage of vascular endothelial cells, which can finally result in hypercoagulability. ${ }^{35-37}$ No PE occurred in the present study, due to a number of possible reasons. Firstly, the patients were examined using lower extremity color Doppler flow imaging within 3 days following surgery, whereby anticoagulant therapy was applied in time after identifying DVT and was continued for at least three months. The early sufficient anticoagulant therapy might reduce the rate of distal DVT spreading and may thereby have prevented the occurrence of PE. However, asymptomatic PE may have been overlooked, 


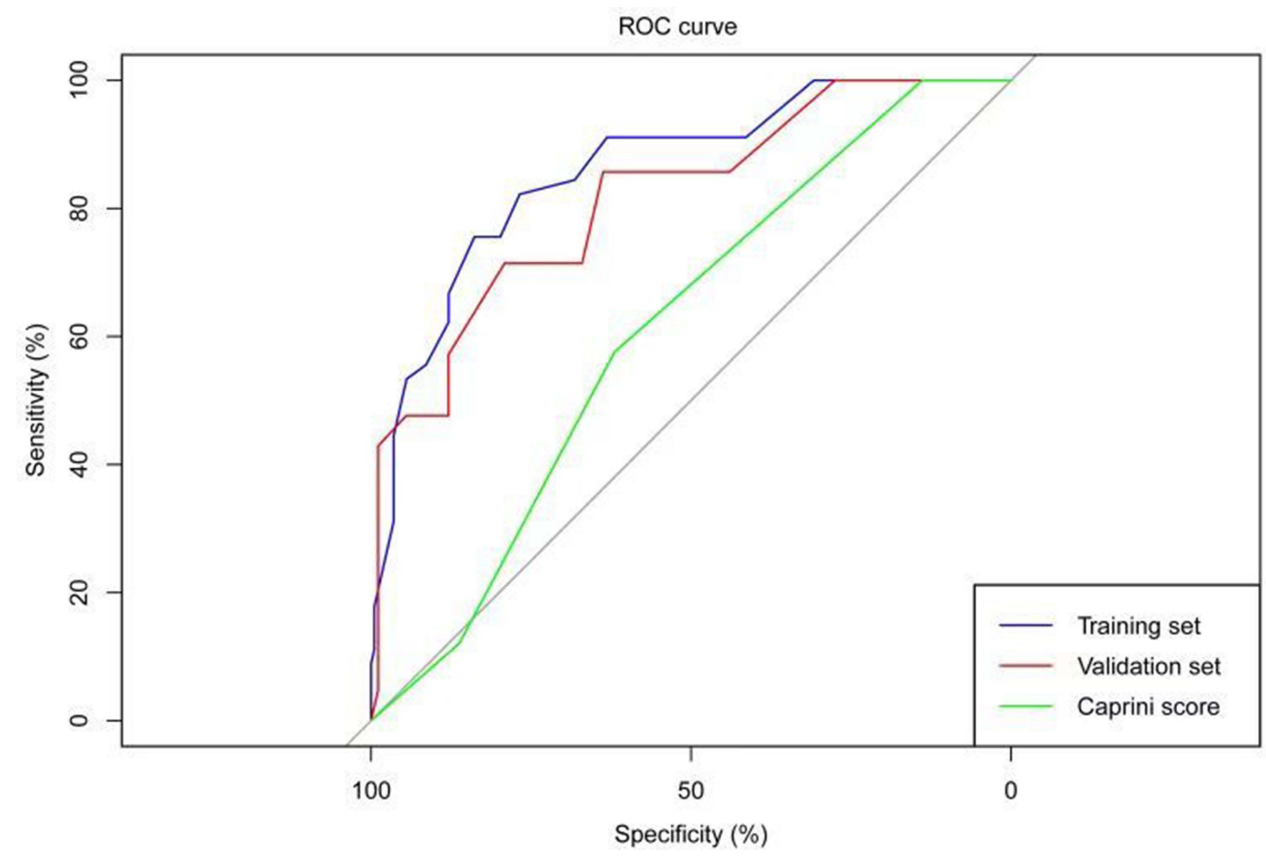

Figure 2 The ROC curves of DVT risk score algorithm and Caprini score.

Abbreviations: ROC, receiver operating characteristic curve; AUC, area under the receiver operating characteristic curve.

and it was reported that peripheral PE was usually found in patients with few or no symptoms. ${ }^{38}$ Prasad and colleagues suggested that anticoagulant therapy had little benefit for peripheral $\mathrm{PE}$ and increased the incidence of adverse events. ${ }^{39}$ In the present study, we found that the rate of disappearance of unilateral distal DVT was higher than that of bilateral distal DVT on the 30th day of anticoagulant therapy ( $92.5 \%$ vs $79.2 \%$ ), while the two DVT locations had similar rates of disappearance on the 90th day of anticoagulant therapy ( $95.0 \%$ vs $95.8 \%)$.

In the present study, age, sex, BMI, comorbidities and OPP were independent risk factors of DVT after LH in HCC patients. Advanced age, high BMI and comorbidities (including diabetes and hypertension) have been reported to be associated with DVT in several studies. ${ }^{40-50}$ However, whether female sex is an independent risk factor for DVT in cancer patients is controversial. Turley and colleagues found the male sex was significantly associated with VTE after hepatic resection. ${ }^{2}$ It was also reported that female sex was a risk factor for VTE in cancer patients ${ }^{34}$ and in chronic liver disease patients. ${ }^{33}$ Naess and colleagues stratified patients by sex and age, which indicated that women between the ages of 20 to 60 had a higher absolute risk of VTE than men, and the VTE risk increased with age. ${ }^{51}$ In the present study, the risk of DVT was significantly higher in women than in men $(p<0.001$, odds ratio $(\mathrm{OR})=13.966)$, which may be due to a number of reasons. Firstly, women were more prone to inactivity due to pain after surgery, which is a significant risk factor for DVT. ${ }^{52}$ Secondly, women had a higher age than men among both the overall patients and the DVT group (overall: $57.4 \pm 9.3$ vs $54.9 \pm 10.9, p=$ 0.032; DVT group: $57.3 \pm 8.5$ vs $54.5 \pm 11.1, p=0.040$ ). In the present study, we firstly found that OPP during LH was significant associated with the development of DVT. During LH, the patients were usually in a reverse Trendelenburg and right oblique position when the right hepatectomy was performed. Due to the change of position and $\mathrm{CO}_{2}$ pneumoperitoneum, the venous return was reduced, resulting in venous stasis in lower extremities, ${ }^{13}$ which is one of the main risk factors for perioperative DVT and PE. ${ }^{14}$

The Caprini score might need to be modified for our study population due to its poor predictive performance. All patients in this study scored $\geq 5$ on the Caprini scale, and were defined as having high risk of DVT, which means that all the patients should receive pharmacological thromboprophylaxis. Well-developed guidelines are available in the West, while routine pharmacological thromboprophylaxis in Asia is more controversial. ${ }^{53}$ Compared to the Western population, the Asian population has a significant lower incidence 
A

Points

Age

Sex

BMI

Comorbidities

Opp

Total Points

DVT Risk

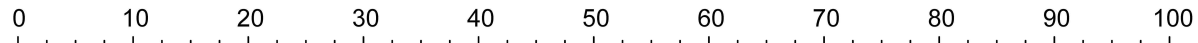

$\geq 60$

$<60$

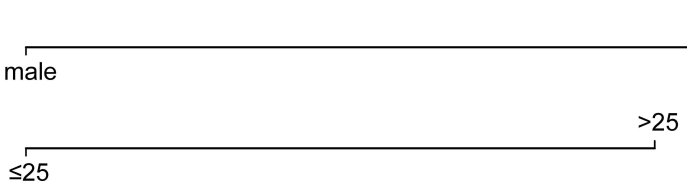

$>25$

yes

no

yes

no

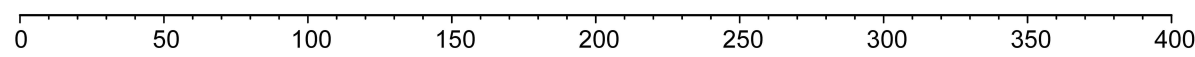

B
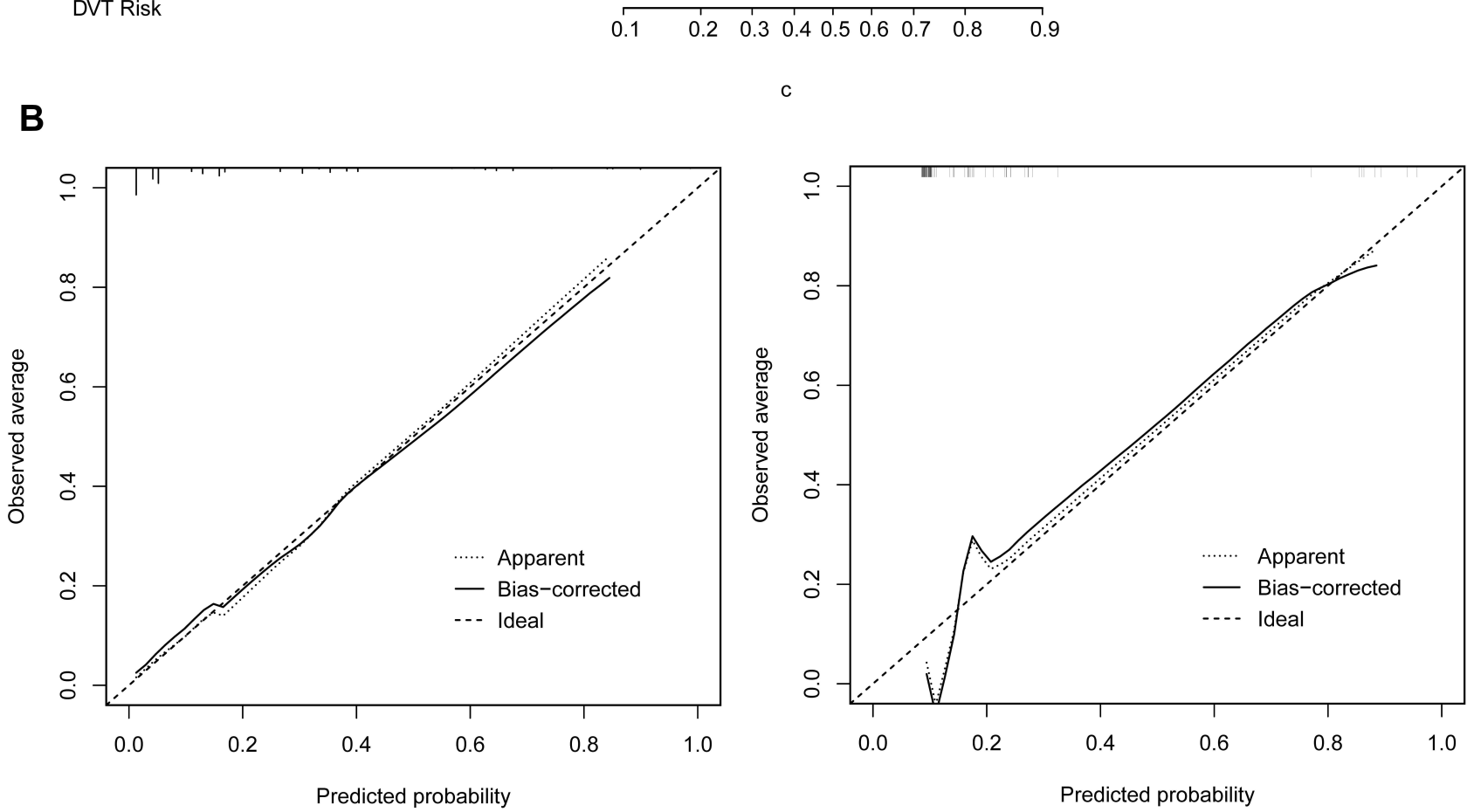

Figure 3 The nomogram based on the DVT risk score algorithm and the calibration curves of the TRN. The nomogram based on DVT risk score algorithm (A), the calibration curves of TRN in the TS (B) and in the VS (C). To use the TRN, the predicted DVT risk is on the scale of 0 to 400 , and a vertical line is drawn upward to the points line to determine the score received for each variable (eg sex female $=100$ points). Then the total score is obtained, which corresponds to a predictive probability of DVT on the predicted incidence line at the bottom of the nomogram.

Abbreviations: TRN, DVT risk nomogram; BMI, body mass index; OPP, operative position; DVT, deep vein thrombosis; TS, training set; VS, validation set.

of VTE, even among subjects who live in the same region, ${ }^{54}$ and physical prophylaxis can be an effective and safe method for VTE prevention, which is preferred by Asian physicians in clinical practice. ${ }^{3,55}$ Accordingly, physical prophylaxis rather than chemoprophylaxis was adopted in our center, and the incidence of DVT was $18.6 \%$, although most thrombus cases presented in the calf veins. However, a published study suggested that the rate of distal DVT spreading to the popliteal vein or higher veins was $17.0 \%$, with up to $5.8 \%$ leading to $\mathrm{PE}$ if no intervention was performed. ${ }^{6}$ What's more, anticoagulation rather than surveillance was recommended for high-risk patients with distal DVT. ${ }^{25,56}$ In our study, all the DVT patients had more than one high 


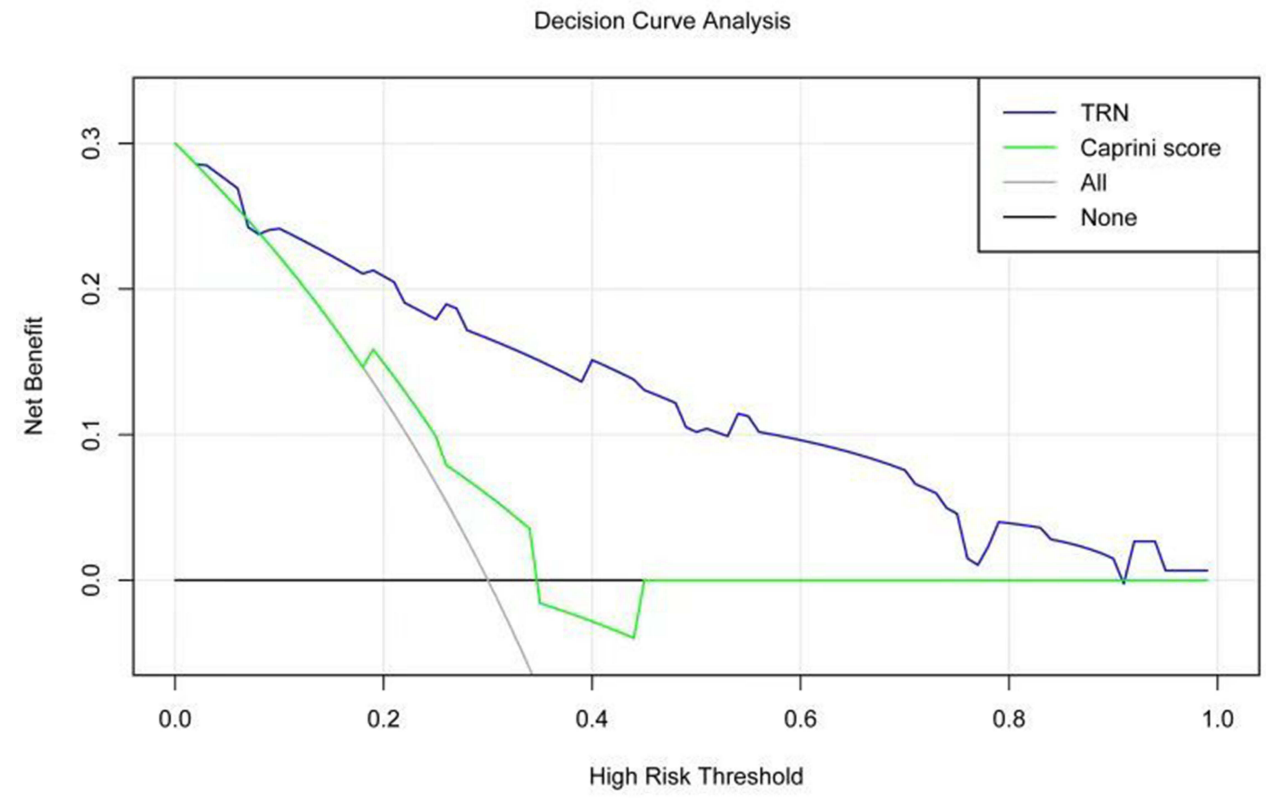

Figure 4 The decision curve analysis for TRN and Caprini score.

Abbreviations: TRN, DVT risk nomogram; DVT, deep vein thrombosis.

risk factor (active cancer, inpatient status, etc.) that supported anticoagulation. In conclusion, there are currently no suitable tools to evaluate the risk of DVT after $\mathrm{LH}$ to guide individualized treatment in $\mathrm{HCC}$ patients. Here, we developed the TRN, which exhibited good performance in predicting the risk of DVT after LH in HCC patients. Furthermore, the decision curve showed that TRN had a better clinical application value than the Caprini score in the study population. Hence, by allowing an accurate evaluation of DVT risk, the TRN might be helpful in guiding the individual treatment with pharmacological thromboprophylaxis in clinical practice.

Finally, there are also a number of limitations to this study. Firstly, this is a retrospective study containing

Table 3 The Cumulative Number of DVT Disappearance

\begin{tabular}{|l|c|c|c|c|}
\hline Variables & $\begin{array}{c}\text { Unilateral } \\
\text { Distal DVT } \\
\text { n (\%) }\end{array}$ & $\begin{array}{c}\text { Bilateral } \\
\text { Distal } \\
\text { DVT } \\
\text { n (\%) }\end{array}$ & $\begin{array}{c}\text { Unilateral } \\
\text { Proximal } \\
\text { DVT } \\
\text { n (\%) }\end{array}$ & $\begin{array}{c}\text { Bilateral } \\
\text { Proximal } \\
\text { DVT } \\
\text { n (\%) }\end{array}$ \\
\hline 0th day* & $40(60.6)$ & $24(36.4)$ & $2(3.0)$ & 0 \\
5th day* & $20(55.6)$ & $\mid \mathrm{I}(38.9)$ & $2(5.5)$ & 0 \\
10th day* & I0(50.0) & $8(40.0)$ & $2(10.0)$ & 0 \\
30th da** & $3(33.3)$ & $5(55.6)$ & $\mathrm{I}(11.1)$ & 0 \\
60th day* & $2(40.0)$ & $2(40.0)$ & $\mathrm{I}(20.0)$ & 0 \\
90th da** & $2(66.7)$ & $\mathrm{I}(33.3)$ & 0 & 0 \\
\hline
\end{tabular}

Note: *Days after anticoagulant therapy. Abbreviation: DVT, deep venous thrombosis. a small sample of 355 HCC patients from a single center, and the predictive performance of TRN needs to be further validated in other centers. Additionally, there was no clear evidence which patients would benefit from the lower extremity color Doppler flow imaging examination, and not all the patients routinely received lower extremity color Doppler flow imaging examination after surgery (Figure 1). As a result, we might have missed some asymptomatic DVT patients. Additionally, no DVT screening was performed before surgery, which might have affected the accuracy of postoperative DVT incidence. In addition, although the lower extremity color Doppler flow imaging examination is recommended as the preferred method for clinically suspected DVT, its accuracy is reduced in the diagnosis of intramuscular venous thrombosis. ${ }^{57}$ Finally, the effects of physical thromboprophylaxis, including intermittent pneumatic compression and early mobilization performed in all patients after surgery, were not explored in the present study.

\section{Conclusions}

The TRN consisting of five independent risk factors could accurately predict the risk of DVT following LH in HCC patients, and thus can help guide individualized pharmacological thromboprophylaxis during the perioperative period of LH. 


\section{Abbreviations}

DVT, deep vein thrombosis; HCC, hepatocellular carcinoma; LH, laparoscopic hepatectomy; TS, training set; VS, validation set; TRN, DVT risk nomogram; BMI, body mass index; AUC, area under the receiver operating characteristic curve; PPV, positive predictive value; NPV, negative predictive value; VTE, venous thromboembolism; PE, pulmonary embolism; NSQIP, national surgical quality improvement program; LMWH, low molecular weight heparin; AIC, Akaike information criterion; H-L, Hosmer-Lemeshow; BMI, body mass index; PLT, platelets; INR, international normalized ratio; MTS, maximum tumor size; BCLC, Barcelona clinic liver cancer; OT, operation time; IT, intraoperative transfusion; OPP, operative position; CI, confidence intervals; OR, odds ratio; ROC, receiver operating characteristic curve.

\section{Data Sharing Statement}

Data are available upon request from the correspondence author, Dr. Yifa Chen or Dr. Wanguang Zhang.

\section{Ethics Approval and Informed Consent}

The written informed consent for data use was obtained from all the patients. This study was approved by the Ethics Committee of Tongji Hospital, Tongji medical college, Huazhong University of Science and Technology.

\section{Acknowledgment}

The authors would like to thank all the surgeons and patients who participated in the study.

\section{Funding}

This study was supported by the National Natural Science Foundation of China (No. 81874149) and the State Key Project on Infectious Diseases of China (No.2018ZX10723204-003-002).

\section{Disclosure}

The authors report no conflicts of interest in this work.

\section{References}

1. Levitan N, Dowlati A, Remick SC, et al. Rates of initial and recurrent thromboembolic disease among patients with malignancy versus those without malignancy. Risk analysis using medicare claims data. Medicine. 1999;78(5):285-291. doi:10.1097/00005792-199909000-00001

2. Turley RS, Reddy SK, Shortell CK, Clary BM, Scarborough JE. Venous thromboembolism after hepatic resection: analysis of 5706 patients. J Gastrointest Surg. 2012;16(9):1705-1714. doi:10.1007/ s11605-012-1939-x
3. Yhim HY, Jang MJ, Bang SM, et al. Incidence of venous thromboembolism following major surgery in Korea: from the health insurance review and assessment service database. J Thromb Haemost. 2014;12(7):1035-1043. doi:10.1111/jth.12611

4. Jang MJ, Bang SM, Oh D. Incidence of venous thromboembolism in Korea: from the health insurance review and assessment service database. J Thromb Haemost. 2011;9(1):85-91. doi:10.1111/j.15387836.2010.04108.x

5. Cheuk BL, Cheung GC, Cheng SW. Epidemiology of venous thromboembolism in a Chinese population. Br J Surg. 2004;91(4):424-428. doi:10.1002/bjs.4454

6. Garry J, Duke A, Labropoulos N. Systematic review of the complications following isolated calf deep vein thrombosis. $\mathrm{Br} J$ Surg. 2016;103(7):789-796. doi:10.1002/bjs. 10152

7. Ejaz A, Spolverato G, Kim Y, et al. Defining incidence and risk factors of venous thromboemolism after hepatectomy. $J$ Gastrointest Surg. 2014;18(6):1116-1124. doi:10.1007/s11605-013-2432-x

8. Lesmana CR, Inggriani S, Cahyadinata L, Lesmana LA. Deep vein thrombosis in patients with advanced liver cirrhosis: a rare condition? Hepatol Int. 2010;4(1):433-438. doi:10.1007/s12072-010-9166-6

9. Scotte F, Rey JB, Launay-Vacher V. Thrombosis, cancer and renal insufficiency: low molecular weight heparin at the crossroads. Support Care Cancer. 2012;20(12):3033-3042. doi:10.1007/s00520012-1590-9

10. Sorensen HT, Mellemkjaer L, Olsen JH, Baron JA. Prognosis of cancers associated with venous thromboembolism. $N$ Engl J Med. 2000;343(25):1846-1850. doi:10.1056/NEJM200012213432504

11. Vivarelli M, Zanello M, Zanfi C, et al. Prophylaxis for venous thromboembolism after resection of hepatocellular carcinoma on cirrhosis: is it necessary? World J Gastroenterol. 2010;16 (17):2146-2150. doi:10.3748/wjg.v16.i17.2146

12. Nguyen KT, Gamblin TC, Geller DA. World review of laparoscopic liver resection-2804 patients. Ann Surg. 2009;250(5):831-841. doi:10.1097/SLA.0b013e3181b0c4df

13. Sharma A, Dahiya D, Kaman L, Saini V, Behera A. Effect of various pneumoperitoneum pressures on femoral vein hemodynamics during laparoscopic cholecystectomy. Updates Surg. 2016;68(2):163-169. doi:10.1007/s13304-015-0344-x

14. Mayol J, Vincent-Hamelin E, Sarmiento JM, et al. Pulmonary embolism following laparoscopic cholecystectomy: report of two cases and review of the literature. Surg Endosc. 1994;8(3):214-217. doi:10.1007/BF00591834

15. Cronin M, Dengler N, Krauss ES, et al. Completion of the updated caprini risk assessment model (2013 version). Clin Appl Thromb Hemost. 2019;25:1076029619838052. doi:10.1177/1076029619838052

16. World Medical Association. World medical association declaration of Helsinki: ethical principles for medical research involving human subjects. JAMA. 2013;310(20):2191-2194.

17. Wang J, Zhao JP, Wang JJ, et al. The impact of bile leakage on long-term prognosis in primary liver cancers after hepatectomy: a Propensity-Score-Matched Study. Asian J Surg. 2020;43(5):603-612. doi:10.1016/j.asjsur.2019.08.009

18. Chen XP, Wu ZD, Huang ZY, Qiu FZ. Use of hepatectomy and splenectomy to treat hepatocellular carcinoma with cirrhotic hypersplenism. Br J Surg. 2005;92(3):334-339. doi:10.1002/bjs.4776

19. Pei Y, Chai S, Zhang Y, Zhang Z, Chen X, Zhang W. Benefits of splenectomy and curative treatments for patients with hepatocellular carcinoma and portal hypertension: a Retrospective Study. $J$ Gastrointest Surg. 2018;23(11):2151-62. doi:10.1007/s11605-0183981-9

20. Righini M, Galanaud JP, Guenneguez H, et al. Anticoagulant therapy for symptomatic calf deep vein thrombosis (CACTUS): a randomised, double-blind, placebo-controlled trial. Lancet Haematol. 2016;3(12):e556-e562. doi:10.1016/S2352-3026(16) 30131-4 
21. Pollack CV, Schreiber D, Goldhaber SZ, et al. Clinical characteristics, management, and outcomes of patients diagnosed with acute pulmonary embolism in the emergency department: initial report of EMPEROR (multicenter emergency medicine pulmonary embolism in the real world registry). J Am Coll Cardiol. 2011;57(6):700-706. doi:10.1016/j.jacc.2010.05.071

22. Miniati M, Prediletto R, Formichi B, et al. Accuracy of clinical assessment in the diagnosis of pulmonary embolism. Am J Respir Crit Care Med. 1999;159(3):864-871. doi:10.1164/ajrccm.159.3.9806130

23. Wells PS, Ginsberg JS, Anderson DR, et al. Use of a clinical model for safe management of patients with suspected pulmonary embolism. Ann Intern Med. 1998;129(12):997-1005. doi:10.7326/ 0003-4819-129-12-199812150-00002

24. Young AM, Marshall A, Thirlwall J, et al. Comparison of an oral factor xa inhibitor with low molecular weight heparin in patients with cancer with venous thromboembolism: results of a randomized trial (SELECT-D). J Clin Oncol. 2018;36(20):2017-2023. doi:10.1200/ JCO.2018.78.8034

25. Kearon C, Akl EA, Ornelas J, et al. Antithrombotic therapy for VTE disease: CHEST guideline and expert panel report. Chest. 2016;149 (2):315-352.

26. Zhou H, Hu Y, Li X, et al. Assessment of the risk of venous thromboembolism in medical inpatients using the padua prediction score and caprini risk assessment model. J Atheroscler Thromb. 2018;25(11):1091-1104. doi:10.5551/jat.43653

27. Wakakura S, Hara F, Fujino T, et al. Comparison of direct oral anticoagulants and warfarin in the treatment of deep venous thrombosis in the chronic phase. Int Heart J. 2018;59(1):126-135. doi:10.1536/ihj.16-482

28. Newhook TE, LaPar DJ, Walters DM, et al. Impact of postoperative venous thromboembolism on postoperative morbidity, mortality, and resource utilization after hepatectomy. Am Surg. 2015;81 (12):1216-1223. doi:10.1177/000313481508101220

29. Mismetti P, Laporte S, Darmon JY, Buchmuller A, Decousus H. Meta-analysis of low molecular weight heparin in the prevention of venous thromboembolism in general surgery. Br J Surg. 2001;88 (7):913-930. doi:10.1046/j.0007-1323.2001.01800.x

30. Tzeng CW, Katz MH, Fleming JB, et al. Risk of venous thromboembolism outweighs post-hepatectomy bleeding complications: analysis of 5651 national surgical quality improvement program patients. HPB. 2012;14(8):506-513. doi:10.1111/j.1477-2574.2012.00479.x

31. Reddy SK, Turley RS, Barbas AS, et al. Post-operative pharmacologic thromboprophylaxis after major hepatectomy: does peripheral venous thromboembolism prevention outweigh bleeding risks? J Gastrointest Surg. 2011;15(9):1602-1610. doi:10.1007/s11605-011-1591-x

32. Sakon M, Maehara Y, Yoshikawa H, Akaza H. Incidence of venous thromboembolism following major abdominal surgery: a Multi-Center, Prospective Epidemiological Study in Japan. J Thromb Haemost. 2006;4(3):581-586. doi:10.1111/j.15387836.2006.01786.x

33. Yang Y, Ng HS, Fong JC, Lee LH, Lee LH. The effect of chronic liver disease on venous thromboembolism among medically managed patients in Singapore General Hospital. Thromb Res. 2015;136 (3):548-551. doi:10.1016/j.thromres.2015.06.033

34. Khorana AA, Francis CW, Culakova E, Kuderer NM, Lyman GH. Frequency, risk factors, and trends for venous thromboembolism among hospitalized cancer patients. Cancer. 2007;110 (10):2339-2346. doi:10.1002/cncr.23062

35. Benni PB, MacLeod D, Ikeda K, Lin HM. A validation method for near-infrared spectroscopy based tissue oximeters for cerebral and somatic tissue oxygen saturation measurements. J Clin Monit Comput. 2018;32(2):269-284. doi:10.1007/s11239-015-1317-0

36. Yu Y, Zhang K, Zhang L, Zong H, Meng L, Han R. Cerebral near-infrared spectroscopy (NIRS) for perioperative monitoring of brain oxygenation in children and adults. Cochrane Database Syst Rev. 2018;1:CD010947. doi:10.1002/14651858.CD010947.pub2
37. Damiani E, Adrario E, Girardis M, et al. Arterial hyperoxia and mortality in critically ill patients: a systematic review and meta-analysis. Crit Care. 2014;18(6):711. doi:10.1186/s13054-0140711-X

38. Yoo HH, Nunes-Nogueira VS, Fortes Villas Boas PJ. Anticoagulant treatment for subsegmental pulmonary embolism. Cochrane Database Syst Rev. 2020;2:CD010222. doi:10.1002/14651858. CD010222.pub4

39. Prasad V, Rho J, Cifu A. The diagnosis and treatment of pulmonary embolism: a metaphor for medicine in the evidence-based medicine era. Arch Intern Med. 2012;172(12):955-958. doi:10.1001/ archinternmed.2012.195

40. Farge D, Frere C, Connors JM, et al. 2019 international clinical practice guidelines for the treatment and prophylaxis of venous thromboembolism in patients with cancer. Lancet Oncol. 2019;20 (10):e566-e581. doi:10.1016/S1470-2045(19)30336-5

41. Bai J, Ding X, Du X, Zhao X, Wang Z, Ma Z. Diabetes is associated with increased risk of venous thromboembolism: a systematic review and meta-analysis. Thromb Res. 2015;135(1):90-95. doi:10.1016/j. thromres.2014.11.003

42. Heit JA, Spencer FA, White RH. The epidemiology of venous thromboembolism. J Thromb Thrombolysis. 2016;41(1):3-14. doi:10.1007/s11239-015-1311-6

43. Gariani K, Mavrakanas T, Combescure C, Perrier A, Marti C. Is diabetes mellitus a risk factor for venous thromboembolism? A systematic review and meta-analysis of case-control and cohort studies. Eur J Intern Med. 2016;28:52-58. doi:10.1016/j. ejim.2015.10.001

44. Roach RE, Cannegieter SC, Lijfering WM. Differential risks in men and women for first and recurrent venous thrombosis: the role of genes and environment. J Thromb Haemost. 2014;12(10):1593-1600. doi:10.1111/jth. 12678

45. Farge D, Bounameaux H, Brenner B, et al. International clinical practice guidelines including guidance for direct oral anticoagulants in the treatment and prophylaxis of venous thromboembolism in patients with cancer. Lancet Oncol. 2016;17(10):e452-e466. doi:10.1016/S1470-2045(16)30369-2

46. Streiff MB, Agnelli G, Connors JM, et al. Guidance for the treatment of deep vein thrombosis and pulmonary embolism. J Thromb Thrombolysis. 2016;41(1):32-67.

47. Zhang Y, Yang Y, Chen W, et al. Hypertension associated with venous thromboembolism in patients with newly diagnosed lung cancer. Sci Rep. 2016;6(1):19603. doi:10.1038/srep19603

48. Holst AG, Jensen G, Prescott E. Risk factors for venous thromboembolism: results from the Copenhagen City Heart Study. Circulation. 2010;121(17):1896-1903. doi:10.1161/CIRCULATIONAHA.109.9 21460

49. Liew NC, Alemany GV, Angchaisuksiri P, et al. Asian venous thromboembolism guidelines: updated recommendations for the prevention of venous thromboembolism. Int Angiol. 2017;36(1):1-20. doi:10.23736/S0392-9590.16.03765-2

50. Kroger K, Moerchel C; Thrombose-Initiative e V. Acute deep vein thrombosis-modern diagnostics and treatment. Der Chirurg. 2019;90 (1):71-84.

51. Naess IA, Christiansen SC, Romundstad P, Cannegieter SC, Rosendaal FR, Hammerstrom J. Incidence and mortality of venous thrombosis: a Population-Based Study. J Thromb Haemost. 2007;5 (4):692-699. doi:10.1111/j.1538-7836.2007.02450.x

52. Geerts WH, Pineo GF, Heit JA, et al. Prevention of venous thromboembolism: the seventh ACCP conference on antithrombotic and thrombolytic therapy. Chest. 2004;126(3 Suppl):338S-400S.

53. Yeo DX, Junnarkar S, Balasubramaniam S, et al. Incidence of venous thromboembolism and its pharmacological prophylaxis in Asian general surgery patients: a systematic review. World J Surg. 2015;39 (1):150-157. 
54. White RH, Keenan CR. Effects of race and ethnicity on the incidence of venous thromboembolism. Thromb Res. 2009;123(Suppl 4):S1117. doi:10.1016/S0049-3848(09)70136-7

55. Kim MH, Jun KW, Hwang JK, et al. Venous thromboembolism following abdominal cancer surgery in the Korean population: incidence and validation of a risk assessment model. Ann Surg Oncol. 2019;26(12):4037-4044. doi:10.1245/s10434-019-07633-z
56. Schellong SM. Distal DVT: worth diagnosing? Yes. J Thromb Haemost. 2007;5(Suppl 1):51-54. doi:10.1111/j.1538-7836.200 7.02490.x

57. Hamper UM, DeJong MR, Scoutt LM. Ultrasound evaluation of the lower extremity veins. Radiol Clin North Am. 2007;45(3):525-547, ix. doi:10.1016/j.rcl.2007.04.013

\section{Publish your work in this journal}

The Journal of Hepatocellular Carcinoma is an international, peerreviewed, open access journal that offers a platform for the dissemination and study of clinical, translational and basic research findings in this rapidly developing field. Development in areas including, but not limited to, epidemiology, vaccination, hepatitis therapy, pathology and molecular tumor classification and prognostication are all considered for publication. The manuscript management system is completely online and includes a very quick and fair peer-review system, which is all easy to use. Visit http://www.dovepress.com/ testimonials.php to read real quotes from published authors. 